Bertrand Bise

\title{
Différents exemples de centre de consultation en Suisse
}

Cet article a pour but de résumer un travail réalisé en 2018, pour l'obtention d'un Certificat of Advanced Studies en évaluation de l'Université de Berne, s'intitulant «Création d'un centre de consultation dans le domaine de l'évaluation en Suisse : présentation de différents exemples dans d'autres domaines » dans le contexte de la création du centre de conseil de la SEVAL. Huit centres de consultation ont été étudiés selon différents critères : la structure, les tâches et la procédure. Ce travail a servi de base pour la conception du Service de conseil de la SEVAL.

Catégories d'articles : Reflets de la pratique

Proposition de citation : Bertrand Bise, Différents exemples de centre de consultation en Suisse, in: LeGes 32 (2021) 3 


\section{Table des matières}

1. Introduction

2. Qu'est-ce qu'un centre de consultation?

3. Analyse des caractéristiques importantes des centres de consultation étudiés

3.1. Taille de la structure

3.2. Prix de l'accès aux centres de consultation

3.3. Formalisation de la procédure

4. Conclusion

\section{Introduction}

[1] Le comité de la SEVAL avait inscrit le développement d'offres pour renforcer la qualité des évaluations et l'assurance qualité dans sa stratégie $2018^{1}$. Cette idée se basait en partie sur deux articles parus peu auparavant dans LeGes : dans leur article traitant des interférences dans le domaine de l'évaluation en Suisse, Pleger et SAgER ${ }^{2}$ ont effectué un sondage auprès des mandataires membres de la SEVAL pour étudier le phénomène de l'influence des mandants sur les mandataires dans le monde de l'évaluation en Suisse. 50\% des répondants au sondage ont annoncé avoir été la cible d'influence, et 10 personnes ont proposé de créer un " point de contact » au sein de la SEVAL afin de pouvoir se faire conseiller dans une telle situation. Certains autres sondés ont en outre indiqué que la SEVAL pourrait avoir un rôle de conseil, de médiateur ou encore de consultant.

[2] Dans le deuxième article de Friedrich, Schlaufer et $\mathrm{MiChel}^{3}$, qui avait pour thème la professionnalisation de l'évaluation en Suisse, les personnes sondées étaient également invitées à donner leur opinion sur les différentes mesures pouvant aider à la professionnalisation. 51\% des membres de la SEVAL ayant répondu au sondage ont déclaré que la SEVAL devrait mettre en place un organe de médiation.

[3] Au niveau international, si bon nombre de sociétés d'évaluation nationales proposent des formations, comme par exemple en France ou en Angleterre, aucune ne propose un service de centre de consultation.

\section{Qu'est-ce qu'un centre de consultation?}

[4] Le terme centre de consultation est à prendre au sens large du terme : il comprend toute forme d'organisation créée afin d'aider, de conseiller et/ou de se renseigner dans un domaine. Ces centres peuvent prendre par exemple la forme d'un Ombudsman, d'un centre de médiation, d'un centre d'arbitrage, d'un centre d'expertise ou encore, comme pour la SEVAL, d'un centre de conseil. Ils partagent cependant tous certaines caractéristiques : une taille donnée (évaluée en équivalent plein temps (EPT)), un prix (ou une gratuité) d'accès ainsi qu'un certain formalisme dans la procédure.

\footnotetext{
Stratégie du comité SEVAL 2018-2021; https ://www.seval.ch/app/uploads/2018/08/SEVAL-Strategie_2018-21def-f.pdf.

2 Pleger, Lyn / SAger, Fritz (2016) : Die Beeinflussung in der Evaluationstätigkeit in der Schweiz und was die Seval dagegen tun kann, in : LeGes 27 (2016) 1, p. 33-49.

3 Friedrich, Verena / Schlaufer, Caroline / Michel, Iris (2018) : Professionalisierung der Evaluation : Ergebnisse
} der Seval-Mitgliederbefragung, in : LeGes 28 (2017) 2, p. 347-367. 


\section{Analyse des caractéristiques importantes des centres de consultation étudiés}

[5] Huit centres de consultation ont été étudiés. Il s'agit de l'Ombudsman des banques suisses, l'office de l'Ombudsman de l'hôtellerie suisse, l'Ombudsman de l'assurance privée et de la SUVA, l'Ombudsman de la branche suisse du voyage, l'organe de conciliation indépendant de la Commission fédérale de la poste (Organe de conciliation PostCom), l'organe de conciliation des télécommunications (ombudscom), le service de médiation des transports publics et médiationBAU (association visant à soutenir les institutions, les entreprises et les personnes privées demandant de l'aide et à prévenir ou résoudre des conflits prenant place dans le monde de la construction et de l'immobilier).

Tableau récapitulatif des différents centres de consultation étudiés

\begin{tabular}{|c|c|c|c|c|}
\hline & Taille de la structure & $\begin{array}{l}\text { Nombre de cas } \\
\text { (dernier chiffre } \\
\text { disponible) })^{4}\end{array}$ & $\begin{array}{l}\text { Prix de } \\
\text { l'accès }\end{array}$ & Procédure \\
\hline $\begin{array}{l}\text { Ombudsman des } \\
\text { banques suisses }\end{array}$ & $\begin{array}{l}\text { Conseil de } \\
\text { fondation }+ \\
\text { Ombudsman }+ \\
\text { collaborateurs }\end{array}$ & 2013 & Gratuit & Formalisée \\
\hline $\begin{array}{l}\text { Office de } \\
\text { l'Ombudsman de } \\
\text { l'hôtellerie suisse }\end{array}$ & $\begin{array}{l}\text { Une personne }(0.3 \\
\text { EPT) }\end{array}$ & $\begin{array}{l}\text { Non } \\
\text { communiqué }\end{array}$ & Gratuit & $\begin{array}{l}\text { Peu } \\
\text { formalisée }\end{array}$ \\
\hline $\begin{array}{l}\text { Ombudsman de } \\
\text { l'assurance privée et } \\
\text { de la SUVA }\end{array}$ & $\begin{array}{l}\text { Conseil de } \\
\text { fondation }+ \\
\text { Ombudsman }+2 \\
\text { représentants }+ \\
\text { collaborateurs }\end{array}$ & 3408 & Gratuit & Formalisée \\
\hline $\begin{array}{l}\text { Ombudsman de la } \\
\text { branche suisse du } \\
\text { voyage }\end{array}$ & $\begin{array}{l}\text { Ombudsman }+2 \\
\text { collaborateurs (total } \\
\text { de } 2 \mathrm{EPT} \text { ) }\end{array}$ & 1254 & Gratuit & Formalisée \\
\hline $\begin{array}{l}\text { Organe de } \\
\text { conciliation de la } \\
\text { PostCom }\end{array}$ & $\begin{array}{l}\text { Un représentant } \\
(\text { environ } 0.2 \mathrm{EPT})+ \\
\text { secrétariat (environ } \\
0.2 \mathrm{EPT} \text { ) }\end{array}$ & 2300 & $20 .-$ & Formalisée \\
\hline $\begin{array}{l}\text { Organe de } \\
\text { conciliation des } \\
\text { télécommunications }\end{array}$ & $\begin{array}{l}\text { Conseil de } \\
\text { fondation }+ \\
\text { Ombudsman }+ \\
\text { collaborateurs }\end{array}$ & 1464 & 20.- & Formalisée \\
\hline $\begin{array}{l}\text { Service de } \\
\text { médiation des } \\
\text { transports publics }\end{array}$ & $\begin{array}{l}\text { Ombudsman }+ \\
\text { représentant } \\
\text { romand }\end{array}$ & 122 & Gratuit & $\begin{array}{l}\text { Peu } \\
\text { formalisée }\end{array}$ \\
\hline médiationBAU & $\begin{array}{l}\text { Plateforme pour } \\
\text { mettre en relation }\end{array}$ & $\begin{array}{l}\text { Non } \\
\text { communiqué }\end{array}$ & $\begin{array}{l}\text { Selon } \\
\text { accord }\end{array}$ & $\begin{array}{l}\text { Selon } \\
\text { accord }\end{array}$ \\
\hline
\end{tabular}




\subsection{Taille de la structure}

[6] La taille des différents centres étudiés est de manière générale corrélée au nombre de cas que ces mêmes centres traitent chaque année. L'exception de l'organe de conciliation de la PostCom s'explique du fait qu'une très grande majorité des cas qui lui sont adressés ne sont pas de sa compétence.

[7] Une grande structure, comme l'Ombudsman de l'assurance privée et de la SUVA, celui de la branche suisse du voyage ou encore l'organe de conciliation des télécommunications, comporte deux avantages : d'une part avoir plus de personnel permet de traiter plus de cas par année, d'autre part il est aussi possible d'être plus réactif et, donc, plus rapide. Il est cependant à signaler que les deux plus petites structures, l'Office de l'Ombudsman de l'hôtellerie suisse et l'organe de conciliation de la PostCom, n'ont pas exprimé avoir de problème lié à la lenteur de la procédure.

[8] Il est important de signaler que la taille de la structure se doit d'être la plus restreinte possible. Bien que le tableau récapitulatif montre que la procédure est la plupart du temps gratuite pour les demandeurs, les structures sont tout de même financées d'une manière ou d'une autre, le plus souvent par l'argent des associations liées au centre de consultation.

\subsection{Prix de l'accès aux centres de consultation}

[9] Le cas particulier de médiationBAU mis à part, tous les centres de consultation ont en commun la presque gratuité de leur accès. Les émoluments exigés par l'organe de conciliation de la PostCom et par l'organe de conciliation des télécommunications remplissent une obligation légale, raison pour laquelle ces organismes demandent un prix qu'ils ont volontairement fixé bas (20 francs).

[10] La gratuité est importante pour que l'accès aux différents centres de consultation soit possible pour le plus grand nombre de personnes. Un émolument, même faible, a tendance à décourager certaines personnes à faire une demande. Le service d'un centre de consultation doit faire le maximum pour être connu de tous et accessible à tout le monde.

\subsection{Formalisation de la procédure}

[11] La plupart des procédures sont très formalisées, mais certaines le sont moins. C'est le cas pour l'Office de l'Ombudsman de l'hôtellerie suisse et pour le Service de médiation des transports publics, qui ont aussi la particularité d'être des petites structures.

[12] Formaliser une procédure comporte certains avantages : une procédure permet à une personne voulant prendre contact avec un centre de consultation, avant de s'engager, de savoir à quoi s'attendre et de savoir également l'investissement (en temps) que cela impliquera. Du côté du centre de consultation, une procédure formalisée permet d'être très transparent sur le processus et sur la manière de fonctionner ainsi que de garantir une certaine égalité de traitement dans chaque cas.

$4 \quad$ Les chiffres ont été mis à jour le 30.06.2021. 
[13] Cependant, une procédure moins formalisée a aussi ses avantages : elle permet de pouvoir analyser au cas par cas chaque demande qui arrive au centre de consultation. Pour le demandeur, c'est une garantie d'être entendu sans forcément devoir fournir un trop gros investissement. Et du côté du centre de consultation, cela permet de s'adapter à chaque cas.

[14] Le choix de la formalisation de la procédure dépendra également beaucoup du domaine dans lequel le centre de consultation agit : il n'est pas étonnant de voir que l'Ombudsman des banques suisses et l'Ombudsman de l'assurance privée et de la SUVA, deux structures importantes et médiatiquement exposées, ont toutes les deux des procédures très formalisées. Cela leur permet par exemple de les mettre quelque peu à l'abris des risques d'inégalité de traitement.

\section{Conclusion}

[15] Cet article a montré tout d'abord que les structures des centres de consultation suisses étudiés ici sont de tailles très différentes. Ce n'est pas surprenant, les différents centres provenant de domaines très hétéroclites et ayant une importance très variable au sein de leurs domaines. La priorité devrait être de faire coïncider la taille de la structure à la demande. Le prix de l'accès aux centres de consultation est en revanche assez homogène : il est gratuit où il peut l'être, et il consiste en un faible émolument lorsqu'une disposition légale l'exige. De manière générale, la gratuité devrait être la solution privilégiée, afin de garantir un accès au centre de consultation au plus grand nombre de personnes. Pour en finir avec les caractéristiques des centres étudiés, leur procédure est plus ou moins formalisée. Si une procédure formalisée a comme avantage conséquent d'être transparente, une procédure moins formalisée permet d'être plus flexible et de procéder au cas par cas. La préférence dépendra du domaine et, probablement, de l'exposition médiatique des différents centres de consultation.

[16] La SEVAL s'est inspiré des centres de consultation préexistants pour créer son Service de conseil, qui a vu le jour en automne 2020. Dans un autre article du dernier numéro, Martin Koci et Reinhard ZweidenR ${ }^{5}$ expliquent la structure qui a été privilégiée, en particulier sa taille restreinte, la gratuité (jusqu'à un certain point) de sa procédure et son formalisme limité.

Bertrand Bise, Chef suppléant de l'unité Projets législatifs I à l'Office fédéral de la justice.

5 Koci, Martin / Zweidler, Reinhard (2021) : Die neue Beratungsstelle der SEVAL stellt sich kurz vor, in : LeGes 32 (2021) 2. 